


\section{RISK OF DEATH PERSISTS FOR YEARS AFTER HIP FRACTURE}

Kristina Åkesson and Anthony D. Woolf

This meta-analysis, including >500 000 patients with hip fracture, shows substantially increased mortality during the first three months and a remaining excess mortality for up to 10 years in both men and women. The study highlights the need to improve fracture management through systematic approaches that will also reduce mortality.

Hip fracture is generally considered to be the most devastating outcome of osteoporosis. In 1990, 1.66 million hip fractures were reported worldwide,a number that is predicted to rise to 6.3 million by 2050. ${ }^{1}$ Low-trauma hip fractures occur mainly in elderly patients at a mean age of 80. The burden of hip fracture management on both the individual and society is substantial, and is estimated to cost US\$21,000 in the first year alone. ${ }^{2}$ This expenditure includes direct fracture treatment costs and social costs owing to functional impairment and increased morbidity. Hip fractures are associated with a high mortality, particularly during the immediate post-fracture years, and is dependent on both the pre-fracture health status of the patient and the post-fracture medical consequences such as infections, cardiovascular events and tromboembolism.

To elucidate excess mortality related to hip fracture, Haentjens et al. ${ }^{3}$ performed a metaanalysis in which the data from patients with hip fractures was stratified by both sex and time. Overall, 22 cohorts of women and 17 cohorts of men over the age of 50 at the time of hip fracture (and with mortality date available) were included — a total of $>500,000$ individual patients from registry-based and case-based studies performed in the USA or Europe. The study included both short term ( $<2$ years) and long term ( $\leq 10$ years) analyses. As expected, initial mortality following hip fracture was high. In women, a five-fold increased risk for allcause mortality during the first 3 months post-fracture was reported, while men had an eightfold increase over the same time period. Excess mortality persisted after hip fracture at each time interval analyzed (1, 2, 5 and 10 years post-fracture). At $\geq 2$ years post-fracture, the relative increase for all-cause mortality in men and women was about two-fold in comparison with the control population — a ratio that was sustained for up to 10 years.

In terms of absolute risk of death, Haentjens et al. ${ }^{3}$ created a model based on survival data from US patients with hip fracture and mortality risk estimates were presented for an 80-year 
old individual with a hip fracture. Their findings suggest that a woman who meets the stated criteria has an absolute annual mortality risk of $8 \%, 11 \%, 18 \%$ and $22 \%$ at 1, 2, 5 and 10 years post- fracture, respectively, whereas a man has a corresponding absolute risk of 18\%, $22 \%, 26 \%$ and $20 \%$.

This study shows that excess mortality following hip fracture is sustained at a steady rate during 2-10 years of follow-up and is similar for men and women. In the short-term ( $\leq 12$ months post-fracture) the high excess mortality slowly decreases, but remains higher in men compared with women over the entire first year. This latter finding is a well-known phenomenon; men with hip fracture are frailer prior to the fracture than women, which inflicts an increased risk in the post-operative and the post-fracture management periods.

Comorbidities associated with frailty and mortality in patients with hip fracture include cardiovascular disease, infection, thromboembolism and recurrence of fractures.

Although the meta-analysis by Haentjens et al. ${ }^{3}$ was a well-conducted systematic study, it has some limitations (as acknowledged by the authors). The studies included in the analysis were performed at different time-periods; the reported short-term mortality results might, therefore, have been influenced by the overall fracture management at the time when the study was performed. Nevertheless, the presented data show a clear consistency despite a range of observation periods. This study highlights the importance of stratifying the data for sex when analyzing data from patients with hip fracture. However, stratification of the data for patient age at the time of fracture would also have been beneficial, since mortality is clearly influenced by the age of patient when a fracture occurs. ${ }^{4}$ Furthermore, the mean age of occurrence of a hip fracture is increasing, and very elderly individuals ( $>90)$ with hip fracture are becoming a proportionally larger subgroup of all patients, albeit with a shorter expected remaining lifetime regardless of fracture. ${ }^{5}$

Haentjens et al. ${ }^{3}$ provide an important contribution to the knowledge concerning patients with hip fracture and their findings might have clinical implications. The need for improved management in such a vulnerable population is clear and guidelines and initiatives have previously attempted to promote such an improvement. ${ }^{6,7}$ The long-term mortality of patients with hip fracture highlights the need to consider factors at the time of fracture, as well as comorbidities, to improve the long-term outcome. The increased risk of further fractures adding to morbidity and mortality is another important consideration, and standardised 
secondary prevention programs should reduce this risk. Nevertheless, by providing a comprehensive account of excess mortality, this study underlines the need for improved care for patients with hip fracture, both prior to surgery and after surgery, to reduce excess mortality.

Department of Orthopedics, Skåne University Hospital, Lund University, Malmö 205 02, Sweden (K. Åkesson). Duchess of Cornwall Centre for Osteoporosis, Department of Rheumatology, Royal Cornwall Hospital, Truro, Cornwall TR1 3LJ, UK (A. D. Woolf).

Correspondence to: K. Åkesson

kristina.akesson@med.lu.se

doi:10.1038/

Competing interests: None

1. Cooper, C., Campion, G. \& Melton, L. J. 3rd Hip fractures in the elderly: a world-wide projection. Osteoporos. Int. 2, 285-289 (1992).

2. Johnell, O. \& Kanis, J. Epidemiology of osteoporotic fractures. Osteoporos. Int. 16 (Suppl. 2), S3-S7 (2005).

3. Haentjens, P. et al. Meta-analysis: excess mortality after hip fracture among older women and men. Ann. Intern. Med. 152, 380-390 (2010).

4. von Friesendorff, M., Besjakov, J. \& Åkesson, K. Long-term survival and fracture risk after hip fracture: a 22year follow-up in women. J. Bone Miner. Res. 23, 1832-1841 (2008).

5. Bergström, U. et al. The hip fracture incidence curve is shifting to the right. Acta Orthop. 80, 520-524 (2009).

6. McLellan, A. R., Gallacher, S. J., Fraser, M. \& McQuillian, C. The fracture liaison service: success of a program for the evaluation and management of patients with osteoporotic fracture. Osteoporos. Int. 14, 1028-1034 (2003).

7. Sander, B., Elliot-Gibson, V., Beaton, D. E., Bogoch, E. R. \& Maetzel, A. A coordinator program in post-fracture osteoporosis management improves outcomes and saves costs. J. Bone Joint Surg. Am. 90, 1197-1205 (2008). 